\title{
Cooperative Distributed Problem Solving for Controlling Semi-Autonomous and Autonomous Oceanographic Sampling Systems - Phase III
}

\author{
D. Richard Blidberg \\ Autonomous Undersea Systems Institute \\ 86 Old Concord Turnpike \\ Lee NH 03824 \\ phone: (603) 868-3221 fax: (603) 868-3283 email: blidberg@ausi.org
}

Contract Number: N000149615009

http://www.ausi.org

\section{LONG TERM GOALS}

The goal of this program is to develop mechanisms for the adaptive control of a distributed system. This capability will provide the means for an Autonomous Oceanographic Sampling Network (AOSN) [1] to effectively control its group of vehicles and instrument platforms (VIPs) in the face of changing mission requirements, a changing pool of available VIPs, and a dynamic environment.

\section{S\&T OBJECTIVES}

This work utilizes AUSI's CADCON simulator [2] and University of Maine's CoDA simulator [3]. The major objectives of this phase of work are to:

\$ Implement a prototype a fleet oriented client for the CADCON Simulator: ASMAC (Autonomous System Monitoring and Control).

\$ Integrate the efforts at University of Maine with the activities at AUSI by connecting the CADCON simulator to the CoDA simulator. The UMaine component of this work was funded independently by ONR/DEPSCoR grant N00014-98-1-0648.

\section{APPROACH}

For the ASMAC fleet controller prototype, the Virtual AUV Mission was selected as the subject for the implementation of a fleet controller. This mission involves using a set of four Solar powered AUVS (SAUVs) that cooperate with each other in order to ensure that there is always one AUV on station recording data at a designated reconnaissance location. A principle property of the envisioned ASMAC tool is its ability to assist the human user in his effort to work out a mission specification, and do so in a manner that does not make operational demands on the vehicles which cannot be met. An additional requirement is that ASMAC be useable by operators that may not have detailed knowledge about the vehicles they are controlling. Thus, the ASMAC tool prompts the user for top level mission parameters only, runs through a large set of mission computations, and presents the expected outcome of such a mission to the user for examination. Encoding this sequence in a graphically oriented interactive loop allows the user to experiment with top level mission concerns such as major way points and expected water currents, while the tool works out the viability of the overall mission based on the expected energy consumption caused by the user's input. 


\section{Report Documentation Page}

Form Approved

OMB No. 0704-0188

Public reporting burden for the collection of information is estimated to average 1 hour per response, including the time for reviewing instructions, searching existing data sources, gathering and maintaining the data needed, and completing and reviewing the collection of information. Send comments regarding this burden estimate or any other aspect of this collection of information,

including suggestions for reducing this burden, to Washington Headquarters Services, Directorate for Information Operations and Reports, 1215 Jefferson Davis Highway, Suite 1204, Arlington

VA 22202-4302. Respondents should be aware that notwithstanding any other provision of law, no person shall be subject to a penalty for failing to comply with a collection of information if it

does not display a currently valid OMB control number.

\begin{tabular}{|c|c|c|}
\hline $\begin{array}{l}\text { 1. REPORT DATE } \\
\mathbf{3 0} \text { SEP } 2002\end{array}$ & 2. REPORT TYPE & $\begin{array}{l}\text { 3. DATES COVERED } \\
\mathbf{0 0 - 0 0 - 2 0 0 2} \text { to } 00-\mathbf{0 0 - 2 0 0 2}\end{array}$ \\
\hline \multirow{3}{*}{\multicolumn{2}{|c|}{$\begin{array}{l}\text { 4. TITLE AND SUBTITLE } \\
\text { Cooperative Distributed Problem Solving for Controlling } \\
\text { Semi-Autonomous and Autonomous Oceanographic Sampling Systems - } \\
\text { Phase III }\end{array}$}} & 5a. CONTRACT NUMBER \\
\hline & & 5b. GRANT NUMBER \\
\hline & & 5c. PROGRAM ELEMENT NUMBER \\
\hline \multirow{3}{*}{\multicolumn{2}{|c|}{ 6. AUTHOR(S) }} & 5d. PROJECT NUMBER \\
\hline & & 5e. TASK NUMBER \\
\hline & & 5f. WORK UNIT NUMBER \\
\hline \multicolumn{2}{|c|}{$\begin{array}{l}\text { 7. PERFORMING ORGANIZATION NAME(S) AND ADDRESS(ES) } \\
\text { Autonomous Undersea Systems Institute,,86 Old Concord } \\
\text { Turnpike,,Lee,,NH, } \mathbf{0 3 8 2 4}\end{array}$} & $\begin{array}{l}\text { 8. PERFORMING ORGANIZATION } \\
\text { REPORT NUMBER }\end{array}$ \\
\hline \multirow{2}{*}{\multicolumn{2}{|c|}{ 9. SPONSORING/MONITORING AGENCY NAME(S) AND ADDRESS(ES) }} & 10. SPONSOR/MONITOR'S ACRONYM(S) \\
\hline & & $\begin{array}{l}\text { 11. SPONSOR/MONITOR'S REPORT } \\
\text { NUMBER(S) }\end{array}$ \\
\hline
\end{tabular}

12. DISTRIBUTION/AVAILABILITY STATEMENT

Approved for public release; distribution unlimited

13. SUPPLEMENTARY NOTES

14. ABSTRACT

The goal of this program is to develop mechanisms for the adaptive control of a distributed system. This capability will provide the means for an Autonomous Oceanographic Sampling Network (AOSN) [1] to effectively control its group of vehicles and instrument platforms (VIPs) in the face of changing mission requirements, a changing pool of available VIPs, and a dynamic environment.

15. SUBJECT TERMS

16. SECURITY CLASSIFICATION OF:

a. REPORT

unclassified b. ABSTRACT unclassified c. THIS PAGE unclassified
17. LIMITATION OF ABSTRACT

Same as Report (SAR)
18. NUMBER OF PAGES

19a. NAME OF RESPONSIBLE PERSON 
Integrating CADCON with CoDA is a challenge, since the simulation technologies are so dissimilar. CoDA is a discrete-event simulator (DES) that models time as discrete, with time between events not being modeled. This has the advantage of focusing the simulation on events that occur and allowing much faster than real-time simulations. CADCON, on the other hand, is a near-continuous-time simulator which models the passing of time at a constant fine-grained level. This has advantages-indeed, is necessary--for simulation of vehicle dynamics and sensors. In addition, the two simulators are written in different languages and run on different machines. CoDA is written both in the CLIPS rule-based language (from NASA) and in Lisp, while CADCON is written in C. Fortunately, CADCON was designed to enable clients to communicate with it over the network, so interfacing between the different languages is relatively straightforward.

UMaine is undertaking the integration of CoDA with CADCON, with technical support as needed from AUSI (e.g., bug fixes for CADCON, questions about CADCON internals, etc.). The approach we are taking involves creating simulated AUV "bodies" (CLOS objects) in the Lisp side of the CoDA simulator to correspond to the simulated AUVs in CADCON. These AUV objects handle all communication with their corresponding AUV in CADCON, including sending commands and receiving telemetry and sensor data. The existing CoDA code can then order the AUV objects to do what needs to be done to carry out the mission, and the objects can ensure that telemetry and sensor data is posted to the appropriate places in the rest of CoDA.

The hardest part is getting the two modes of time modeling to work together. Our approach leaves the DES portion of CoDA intact for when it is desirable to run without CADCON and for handling events that are useful for simulation experiments (e.g., to simulate a vehicle failure at a particular time, etc.). However, much of what is now done by the DES will be done by communication with CADCON. For example, currently, movement of an AUV to a location is done by determining how long the AUV should take to arrive there, then posting an event for that time asserting that the AUV is there. In the combined system, this will be done by sending the appropriate movement command to CADCON via the AUV object in question. When the AUV arrives at the location, that information will be posted by the AUV object to CoDA's working memory, which will then trigger whatever rules need to fire in response.

In addition, CoDA is being modified to give it the ability to initiate sessions with CADCON, add or delete AUVs from the simulation, etc., so that it can control simulation experiments. CoDA's simulation of the work phase protocol [3] is also being fleshed out to allow reasonable missions to be run with the combined simulator.

\section{WORK COMPLETED}

Solar recharged AUVs will necessarily be very concerned about their energy budgets, more so than "standard" AUVs. This is an ideal area where an ASMAC tool can assist users who may not be aware of such nuances. In this effort, the details of SAUV energy consumption (for example: communication costs, transit costs, recharging expectations, data collection costs, water current effects) were implemented as a set of equations within a set of interconnected Lotus spreadsheets. After experimentation and adjustment, the equations were then re-implemented in the form of a programming library. This library was implemented in platform neutral $\mathrm{C}$ and is called the Virtual AUV Mission Planning Assistant (VAMPA) library. This forms the foundation for construction of an ASMAC tool that assists the user in developing viable multiple SAUV missions. 
The architecture for ASMAC has been designed and is composed of these major components: the user interface, a master data table, and a command generator. The user interface allows the user to configure, monitor and modify the operation of the SAUV systems. The data table contains all data configured and computed for each participant in the mission. The command generator is activated by the user once the user is satisfied with the plan he/she has configured. The VAMPA library specifically implements the data table component of ASMAC, plus most of the computation functions for it. It also implements select portions of the user interface component. The command generator will be filled out in future work.

Substantial work has been completed on integrating CoDA and CADCON. The AUV objects have been created and tested with CADCON. Design of the work phase protocols and other rules has been done, and implementation is beginning. Design of the modifications to CoDA has been done to match its DES to CADCON's continuous time mode.

\section{RESULTS}

The energy costs for the operation of a Solar powered AUV have been codified to a fine degree of detail. The resulting knowledge has been implemented in the form of a VAMPA programming library suitable for linking against other applications software. A prototypical ASMAC has been implemented, which exercises the VAMPA library.

\section{IMPACT/APPLICATIONS}

This work is most directly applicable to AOSNs and similar systems for characterizing volumes of the ocean. This would include, for example, oceanographic systems as well as those designed for cooperative mine hunting and other missions of interest to the Navy. Our approach provides the infrastructure for experimenting with AOSN participants communicating with each other using the Internet as the connection medium. As AOSNs are a particular example of cooperative distributed problem solving (CDPS) systems, our work on organization and reorganization will be useful to researchers in the field of distributed AI.

\section{TRANSITIONS}

Technology Systems, Inc. (Wiscasset, Maine, http://www.simworks.com/) is using CADCON in their development of a network communications stack for AUV networking (called AUSNet). Specifically, they are using the CADCON client AUVSim's composite behavior mechanism to implement their particular set of multiple agent missions, the same mechanism used to implement our Virtual AUV Mission.

When completed, the CADCON/CoDA combination will be used by the CoDA research group (ONR grant N0001-14-98-1-0648) as well as other research groups at UMaine. For example, the research group working on informativeness in multi-agent communication can make use of this combined simulator. The Orca research group (ONR grant N00014-00-1-0614) intends to make use of at least the AUV objects to allow the Orca AUV mission controller to control simulated AUVs in CADCON, and in the future, Orca will be used as the controller for some of the AUVs in the CoDA work as well. 


\section{RELATED PROJECTS}

This work relates to the UUV Common Control Language (CCL) [4,5] project (ONR grant N0001400WX20837) of Christine Duarte. This project seeks to develop a CCL that will facilitate effective operator to agent and agent to agent communications in the fleet context. The output of a fully implemented ASMAC tool will be in the vocabulary of just such a language.

\section{REFERENCES}

[1] Tom B. Curtin, James G. Bellingham, J. Catipovic, and D. Webb. "Autonomous Oceanographic Sampling Networks". Oceanography, 6(3), 1993.

[2] Steven G. Chappell and Rick J. Komerska. "An Environment for High-Level Multiple AUV Simulation and Communication”. In Underwater Intervention, January, 2001.

[3] Roy M. Turner and Elise H. Turner. "A Two-level, Protocol-based Approach to Controlling Autonomous Oceanographic Sampling Networks". In the IEEE Journal of Oceanic Engineering special issue on autonomous ocean sampling networks, vol. 26, no. 4, pp. 654-666, October, 2001.

[4] Christine N. Duarte and Barry B. Werger. "Defining a Common Control Language for Multiple Autonomous Vehicle Operations". Proceedings of Oceans 2000, September 11-14, 2000, Providence, RI.

[5] Rick J. Komerska, Steven G. Chappell, Liang Peng, and D. Richard Blidberg. "Generic Behaviors as an Interface for Standard AUV Command and Monitoring Language". AUSI Technical Report 9904-01.

\section{PUBLICATIONS}

Charles Benton, James Kenney, Steven G. Chappell and D. Richard Blidberg. Autonomous Undersea Systems Network (AUSNET): Development Status Update. In Proceedings of Oceans 02, October 2002.

Robert Nitzel, Chuck Benton, Steven G. Chappell and D. Richard Blidberg. Exploiting Dynamic Source Routing to Enable Undersea Networking Over and Ad-Hoc Topology. In Underwater Technology '02, Tokyo Japan, April, 2002. 